\title{
Recurrent Rash Secondary to Shiitake Dermatitis
}

\author{
Nguyen $\mathrm{L}^{1}$ and Prodanovic $\mathrm{E}^{2 *}$ \\ ${ }^{1}$ Doctor of Medicine Program, Eastern Virginia Medical \\ School, USA \\ ${ }^{2}$ Texas Skin Center, Houston, Texas, USA \\ *Corresponding author: Prodanovic E, Texas Skin \\ Center, 4101 Greenbriar Drive, Suite \#305, Houston, \\ Texas 77098, USA
}

Received: March 30, 2021; Accepted: April 14, 2021; Published: April 21, 2021

\section{Introduction}

Shiitake dermatitis is a cutaneous reaction from ingesting shiitake mushrooms, which contain a polysaccharide, lentinan that is hypothesized to cause the inflammatory response. The characteristic presentation is pruritic plaques in a linear or flagellate pattern that resembles marks from a whip. Shiitake dermatitis can be distinguished from other similar cutaneous reactions through history and histology. It is typically self-limiting and treatment focuses on symptom management. Since its first description in 1977, shiitake dermatitis has been documented in multiple patients [1]. The following case report is of an individual initially assumed to have an adverse drug reaction and upon a repeat occurrence, was found to have shiitake dermatitis.

\section{Case Presentation}

The patient was a 56-year-old, morbidly obese female with a history of insulin-dependent diabetes mellitus, hypertension, and chronic pain. For a recent upper gastrointestinal endoscopy, she received propofol and in February 2019, she presented with a diffuse erythematous rash. The rash began from the chest and spread to the extremities, with reported pruritus, pain, and fever. The palms and soles were relatively spared. Following a febrile episode, she was admitted to the emergency department for a period of one week. White blood count was elevated with a high of $17.5 \times 10^{\%} / \mathrm{L}$ and lymphocyte percentage was decreased with a low of $17 \%$. Hemoglobin and hematocrit decreased to $9.9 \mathrm{~g} / \mathrm{dL}$ and $30.4 \%$, respectively. The rash was diagnosed as a drug eruption and hydrochlorothiazide was removed from the patient's medications.

In March 2019, the patient experienced a recurrence of symptoms. The trunk and extremities were covered in pruritic linear papules and suggestive of a flagellate pattern (Figure 1).

A more focused history revealed that the patient ingested mushrooms on a regular basis. The previous day, she consumed a mushroom blend with shiitake mushrooms. A punch biopsy of the right breast was performed. The biopsy results included superficial and mid-dermal perivascular and interstitial lymphocytic dermatitis with eosinophils (Figure 2). Interface vacuolopathy, or vacuolar changes of the basal layer, can also be visualized.

The diagnosis of shiitake dermatitis was supported. The patient was advised to avoid shiitake mushrooms and prescribed a topical
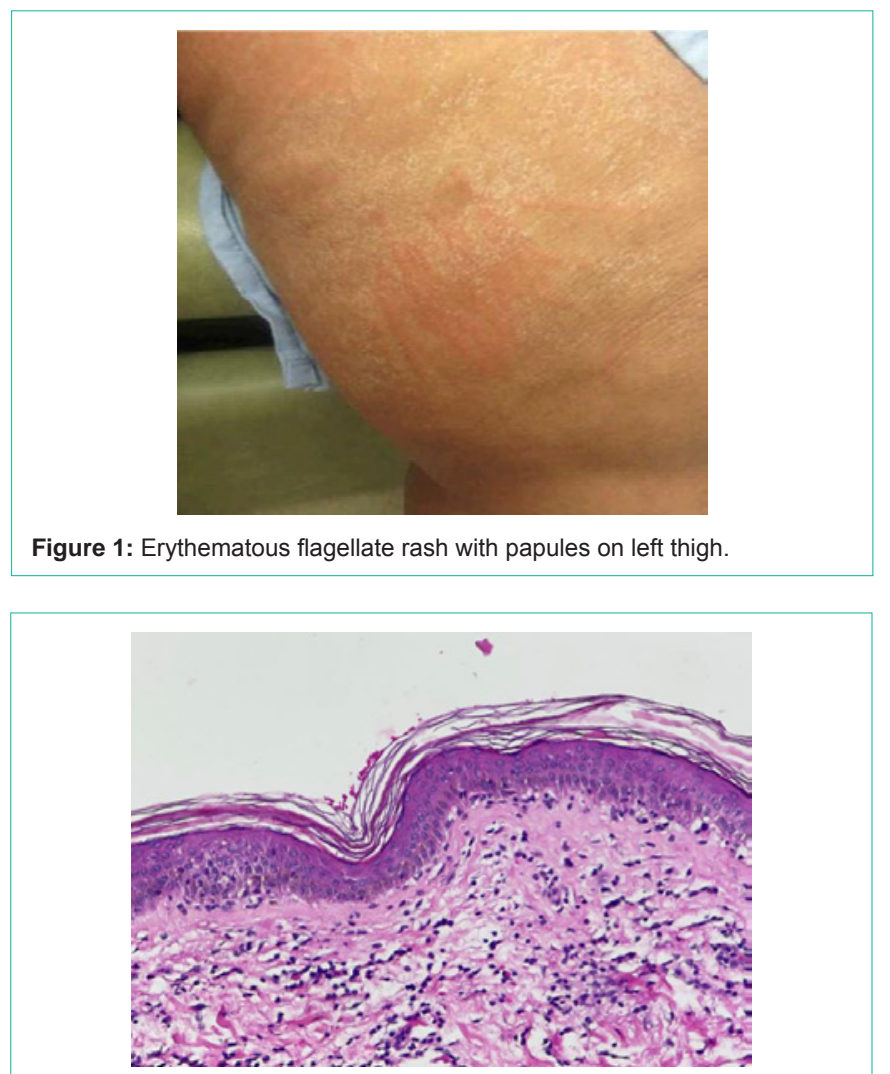

Figure 2: Punch biopsy from right breast. H\&E, 200x.

steroid and prednisone taper for symptom management.

\section{Discussion}

Shiitake dermatitis is a cutaneous reaction that results from eating shiitake mushrooms (Lentinus edodes) [1]. The first cases were reported in 1977, in Japanese patients who had skin eruptions after eating raw or undercooked shiitake mushrooms. Cases also coincided with the harvesting times of shiitake mushrooms [1]. With global consumption of shiitake mushrooms, shiitake dermatitis has been reported in other parts of the world [2].

Shiitake dermatitis presents as linear, erythematous, pruritic plaques or papules. The linear pattern is also referred to as flagellate. Typically, shiitake dermatitis appears on the back, chest, and 
abdomen. The limbs, face, and neck can be involved in descending frequency [3]. Onset typically occurs 24 to 48 hours after ingesting shiitake mushrooms [2], and the average duration of symptoms without treatment is 13 days [3].

The underlying pathophysiology is unknown. The main component of shiitake mushroom that contributes to the reaction is lentinan, which is a polysaccharide in the cell walls of shiitake mushrooms. It has been hypothesized that in shiitake dermatitis, lentinan induces an inflammatory reaction. The proposed mechanism begins with lentinan binding to MHC I on macrophages, which travel to lymph nodes and present lentinan to CD8 T lymphocytes. The $\mathrm{T}$ lymphocytes secrete interferon- $\gamma$, tumor necrosis factor- $\alpha$, and interleukin-2. The overall effect is vasodilation and inflammation. Lentinan is also denatured by heat; shiitake dermatitis can be found after consuming raw or undercooked mushrooms [3].

Histology shows spongiosis and perivascular infiltration of lymphocytes with neutrophilia and eosinophilia. Parakeratosis, interface dermatitis, and focal keratinocyte apoptosis can also be observed4. However, histology alone does not provide a specific diagnosis of shiitake dermatitis.

The differential diagnosis includes drug reactions to bleomycin and trastuzumab, phytophotodermatitis, and dermatographism [2]. Reviewing and confirming the patient's current medications should determine whether drug reactions are likely. A reaction to bleomycin also results in hyperpigmentation of the basal layer [4]. Phytophotodermatitis is a condition in which a cutaneous reaction is induced by contact with juice or sap from certain plants, followed by exposure to sunlight [5]. The reaction is characterized by a bullous or vesicular appearance in areas exposed to the plants
[2]. Dermatographism is a condition in which stroking on skin causes erythema and formation of papules and plaques. However, the reaction recedes within minutes to hours, whereas in shiitake dermatitis, the flagellate pattern lasts for days [2].

Skin tests are not reliable in diagnosing shiitake dermatitis, with patch and prick testing both having inconsistent results [3]. A thorough history is key to diagnosing shiitake dermatitis, along with ruling out other conditions with similar flagellate patterns.

Shiitake dermatitis is self-limited. Treatment is based on management of symptoms. Topical corticosteroids or oral antihistamines are used to reduce inflammation and pruritus [3]. Patients should be advised to fully cook shiitake mushrooms [1] and avoid direct contact during preparation with gloves and other protective equipment [2].

\section{References}

1. Nakamura T. Shiitake (Lentinus edodes) dermatitis. Contact Dermatitis. 1992; 27: 65-70.

2. Stephany MP, Chung S, Handler MZ, Handler NS, Handler GA, Schwartz RA. Shiitake Mushroom Dermatitis: A Review. Am J Clin Dermatol. 2016; 17: 485-489

3. Nguyen AH, Gonzaga MI, Lim VM, Adler MJ, Mitkov MV, Cappel MA. Clinical features of shiitake dermatitis: a systematic review. Int J Dermatol. 2017; 56: 610-616.

4. Ching D, Wood BA, Tiwari S, Chan J, Harvey NT. Histological Features of Flagellate Erythema. Am J Dermatopathol. 2019; 41: 410-421.

5. dos Reis VM. Dermatosis due to plants (phytodermatosis). An Bras Dermatol. 2010; 85: 479-489. 